\title{
Discovery of Repetitive Optical Variation Patterns from the Accretion Disk During the 2015 Outbursts of the Black Hole X-Ray Binary V404 Cyg
}

\author{
Mariko Kimura $^{* a}$, Keisuke Isogai ${ }^{a}$, Taichi Kato ${ }^{a}$, Yoshihiro Ueda ${ }^{a}$, Satoshi Nakahira ${ }^{b}$, \\ Megumi Shidatsu $^{c}$, Teruaki Enoto ${ }^{a, d}$, Takafumi Hori ${ }^{a}$, Daisaku Nogami ${ }^{a}$, Colin \\ Littlefield $^{e}$, Ryoko Ishioka ${ }^{f}$, Elena P. Pavlenko ${ }^{g}$, Alexei S. Pozanenko ${ }^{h, i}$, Akira \\ Imada $^{j}$ \& Makoto Uemura ${ }^{k}$ \\ a Department of Astronomy, Graduate School of Science, Kyoto University, Oiwakecho, \\ Kitashirakawa, Sakyo-ku, Kyoto 606-8502, Japan \\ ${ }^{b}$ JEM Mission Operations and Integration Center, Human Spaceflight Technology Directorate, \\ Japan Aerospace Exploration Agency, 2-1-1 \\ Sengen, Tsukuba, Ibaraki 305-8505, Japan \\ ${ }^{c}$ MAXI team, RIKEN, 2-1 Hirosawa, Wako, Saitama 351-0198, Japan \\ ${ }^{d}$ The Hakubi Center for Advanced Research, Kyoto University, Kyoto 606-8302, Japan \\ ${ }^{e}$ Astronomy Department, Wesleyan University, Middletown, CT 06459 USA \\ ${ }^{f}$ Institute of Astronomy and Astrophysics, Academia Sinica, $11 \mathrm{~F}$ of Astronomy-Mathematics \\ Building, AS/NTU. No.1, Sec. 4, Roosevelt Rd, Taipei 10617, Taiwan (R.O.C.) \\ ${ }^{g}$ Crimean Astrophysical Observatory, 298409, Nauchny, Republic of Crimea \\ ${ }^{h}$ Space Research Institute, Russian Academy of Sciences, 117997 Moscow, Russia \\ ${ }^{i}$ National Research Nuclear University MEPhI (Moscow Engineering Physics Institute), \\ Moscow, Russia \\ ${ }^{j}$ Kwasan and Hida Observatories, Kyoto University, Kitakazan-Ohmine-cho, Yamashina-ku, \\ Kyoto, Kyoto 607-8471, Japan \\ ${ }^{k}$ Hiroshima Astrophysical Science Center, Hiroshima University, Kagamiyama 1-3-1, \\ Higashi-Hiroshima, Hiroshima 739-8526, Japan \\ E-mail: mkimuradkusastro.kyoto-u.ac.jp
}

We report on multi-color optical photometry in the 2015 outbursts of V404 Cygni, an X-ray transient. This system showed optical oscillations on timescales of $100 \mathrm{sec}$ to 2.5 hours in these outbursts, and they were correlated with the simultaneous X-ray variations. On the basis of the $\sim 1$-min optical delays against X-rays and multi-wavelength SED analyses, the optical variability in this system was likely caused by X-ray reprocessing at the outer accretion disk. We also find that some repetitive optical oscillations can occur at mass accretion rates $>10$ times lower than previously thought. Although the mechanism of this kind of variations is still unclear, we suggest that the lack of sustained mass accretion, which would be induced in long-period systems, may be a key condition.

Accretion Processes in Cosmic Sources - APCS2016-

5-10 September 2016,

Saint Petersburg, Russia

${ }^{*}$ Speaker. 


\section{Introduction}

Low mass X-ray binaries (LMXBs) are close binary systems composed of a black hole or a neutron star (the primary) and a typical low-mass main-sequence star (the secondary) [四]. The secondary star fills its Roche lobe and its matter is attracted to the primary star via the Roche-lobe overflow. The matter forms an accretion disk around the primary star and accretes on it through the disk.

Transient LMXBs show sporadic outbursts lasting for dozens of days up to several years in $\mathrm{X}$-rays and optical lights. The interval between their outbursts ranges from several months to tens of years [46, 4]]. In their typical outbursts, their X-ray flux rapidly increase by more than two orders of magnitudes for several days and slowly decrease for dozens of months. The luminosity is $\sim 10^{30-33}$ [erg/s] in quiescence and $\sim 10^{37-39}$ [erg/s] in outbursts [B8]]. The X-ray and optical outburst light curves of transient LMXBs were classified into five types by their morphology with 49 samples [ []]. The most popular outburst is characterized by a fast rise followed by an exponential decay (see also [四]). It is called "Fast Rise Exponential Decay (FRED)-type outburst". The other types are triangle, short or long plateau, variable decay and multiple peak.

The outbursts in transient LMXBs are considered to be caused by thermal-viscous instability due to partial ionization of hydrogen in accretion disks [144, [9]. Under the instability, the state of the disk alternates between cool state in which the transferred mass from the secondary accumulates in the disk and hot state in which the matter accretes onto the primary. This disk instability model was established to explain the outbursts in dwarf novae. (Dwarf novae are close binary systems similar to transient LMXBs, but the primary star is a white dwarf.) As for transient LMXBs, in addition to the disk instability model, the effect of irradiation and evaporation of the inner disk in quiescence is also important to explain the cycles and amplitudes of the outbursts [ [8].

V404 Cyg is a transient LMXB that has a $9 \mathrm{M}_{\odot}$ black hole [ए6] and a $0.7 \mathrm{M}_{\odot}$ companion star

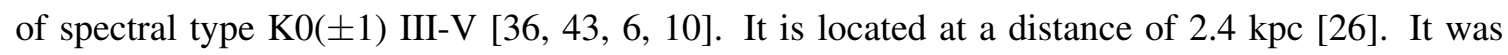
originally discovered as a nova in 1938 and in 1989, identified as X-ray nova by GINGA satellite [23]. During the 1989 outburst, short-term X-ray variability was observed [48]. On June 15th in 2015, it underwent a short outburst after 26 years of quiescence and showed large flares at radio

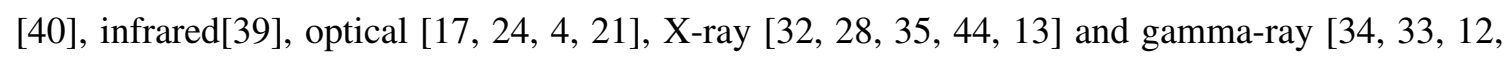
[2]. Some optical spectroscopic observations in the June outburst detected the evidence of outer accretion disk wind [27, [1]]. Additionally, after about 6 months, this object entered the second outburst in 2015. We present our photometric observations in the two outbursts in 2015.

\section{Observations}

Immediately after the detection by Swift/BAT on June 15.77197 UT, the VSNET collaboration team [15]] that we conduct started a worldwide photometric campaign of V404 Cyg in the June outburst. We carried out time-resolved CCD photometry at 26 sites via the VSNET team in the June outburst and 17 sites in the December outburst. The list of sites and telescopes are shown in ED Table 1 in [ㅍ] ]. We also used the data downloaded from the AAVSO archive ${ }^{1}$. The logs of photometric observations in $B, V, R_{\mathrm{C}}$ and $I_{\mathrm{C}}$ bands and with clear filter in the June outburst are

\footnotetext{
${ }^{1}<$ http://www.aavso.org/data/download/>
} 
displayed in ED Table 2 in [ㅁ] ]. The exposure times were 15-540 s. We corrected for bias and flatfielding in the usual manner, and performed standard aperture photometry. The observers except for TAOS [20] used standard filters. All of the observation times were converted to barycentric Julian date (BJD). The constancy of the comparison star was checked by nearby stars in the same images. In the June outburst, we measured magnitudes of V404 Cyg relative to local comparison stars whose magnitudes were measured by A. Henden (sequence 15167RN). They can be derived from the AAVSO Variable Star Database ${ }^{2}$. We applied small zero-point corrections to some observers' measurements.

\section{Results}

\subsection{Overall Optical Light Curves}

V404 Cyg showed slow rise and rapid decay in the June and December outbursts. This trend is rare among the outbursts in LMXBs. The overall optical light curves in the June outburst are displayed in Figure $\square$. The outburst durations were about two weeks. This system showed largeamplitude and short-term optical variations with amplitudes of $\sim 0.1-2.5$ mag on timescales of $\sim 5$ min-2.5 hours throughout the two outburst in 2015. The comparison with the overall X-ray light curves in the June outburst is represented in ED Figure 1 of [ए7] and Figure 1 in [ए8]. The overall trends at optical wavelengths were similar to the X-ray ones in both of the June and December outbursts.
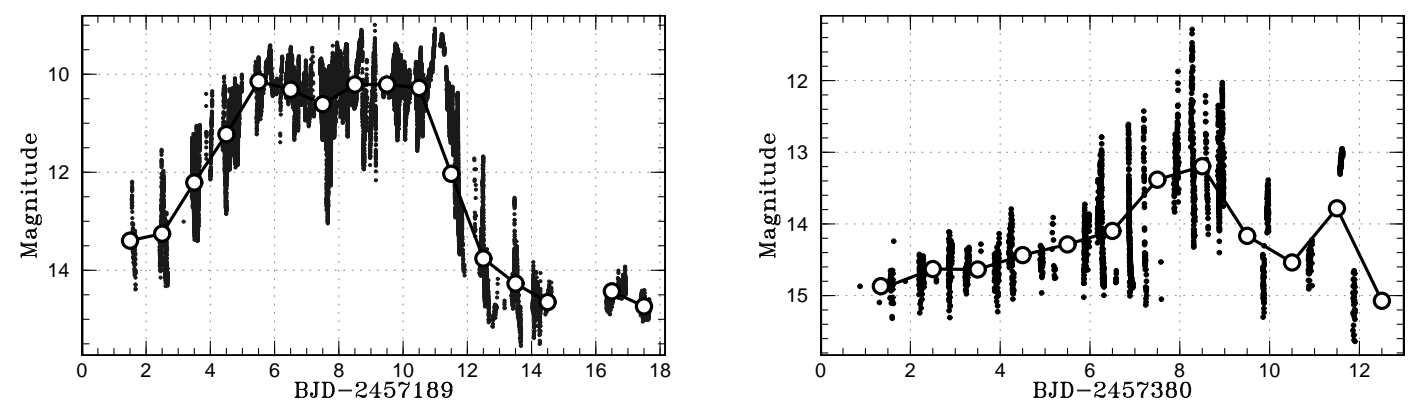

Figure 1: Overall multi-color light curves in the $I_{C}$ band during the 2015 outbursts of V404 Cyg. The left and right panel represent the June and December outbursts, respectively. The open circles are the averaged magnitudes per 1 day. The observational data are derived from those in [ए7, ए8].

\subsection{Short-term Optical Variations}

We detected rapid optical variations with repetitive patterns in the June outburst for the first time among the outbursts in LMXBs. The variations that we observed can be divided into two characteristic classes: (1) dip-type oscillations (repetitions of a gradual rise followed by a sudden dip, sometimes with accompanying spikes on timescales of $\sim 30$ min- 2.5 hours) and (2) heartbeattype oscillations (repetitive small spikes with short periods of $\sim 5 \mathrm{~min}$ ). The two examples are displayed in Figure $\square$. In particular, the dip-type oscillations were observed from the beginning

\footnotetext{
${ }^{2}<$ http://www.aavso.org/vsp $>$
} 
to the end of the outburst. Although rapid optical variations have been detected in the black hole binary V4641 Sgr, the variations are stochastic with no indication of regular patterns [42]. The regular variations that we found in V404 Cyg at optical wavelengths in the June outburst were similar in shape to those in GRS 1915+105 [Q], though the interval between dips is about 5 times longer in V404 Cyg than in GRS 1915+105. Also in the 2015 December outburst, the rapid optical variations were observed, but we did not confirm if they are repetitive variations possibly due to the sparse data [18]].
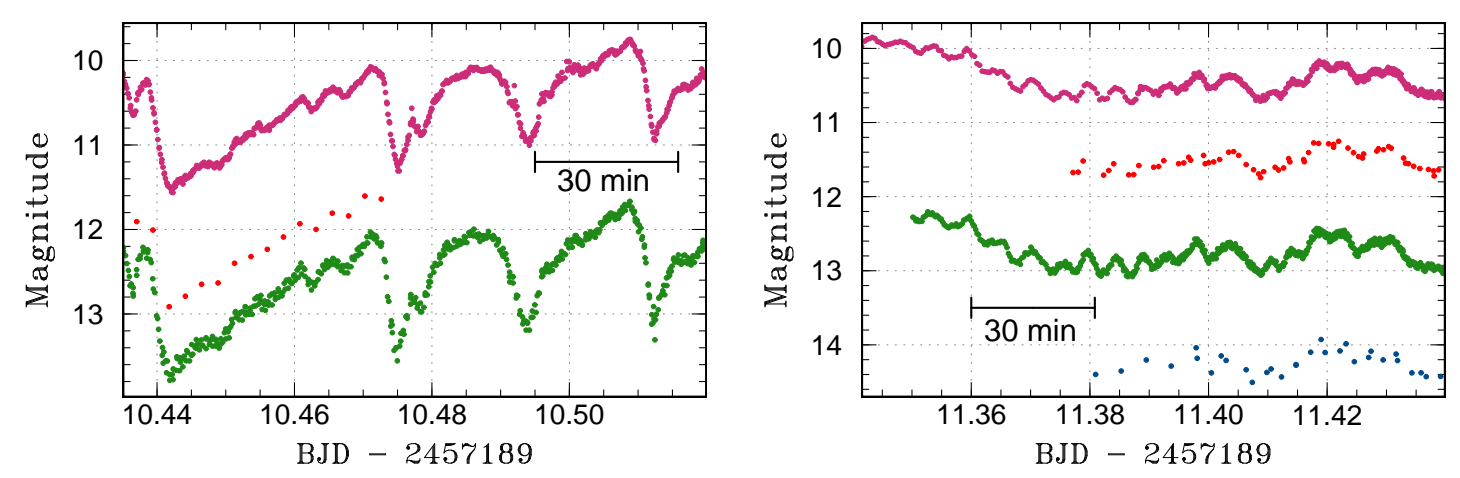

Figure 2: Repetitive optical variations in the June outburst in $B, V R_{\mathrm{C}}$ and $I_{\mathrm{C}}$ bands. Left: an example of dip-type oscillations. Right: an example of heartbeat-type oscillations. The blue, green, red and pink dots represent the light curves in $B, V R_{\mathrm{C}}$ and $I_{\mathrm{C}}$ bands. The observational data are derived from those in [ए]].

\subsection{Comparison with X-ray Variations}

Using the X-ray data took by INTEGRAL IBIS/ISGRI, we compared simultaneous optical and X-ray light curves in the two 2015 outbursts. We give some examples in the June outburst in Figure [3. When both X-ray and optical data showed strong short-term variations, the correlations were generally significant, although the X-ray flux variations are much larger than the optical ones. The significant correlation indicates that both X-ray and optical observations recorded the same phenomena. Spectral analyses of the simultaneous X-ray data in the 2015 June outburst showed that there was no tendency for increased absorption when the X-ray flux decreased, suggesting that these dips do not originate from absorption (This is described in Sec. 3 in Methods of [ए]]). In some epochs, we found evidence for heavy obscuration as found in the GINGA data during the 1989 outburst[[48]; however this is not related to the dip-type variations. We thus considered that the dup-type fluctuations directly reflect variations in radiation from the accretion disk. In the June outburst, we also performed detailed analyses of the simultaneous multi-wavelength spectral energy distribution (SED), and they demonstrated that the majority of the optical flux was likely produced by reprocessing of X-ray irradiation in the disk (This is described in Sec. 8 in Methods of [ㅍ]). Additionally, the delay of optical variations to the X-ray ones was $\sim 1 \mathrm{~min}$ in that outburst, and this supports the $\mathrm{X}$-ray irradiation is the origin of the optical variations. Also in the December outburst, we found $\sim 30$-s optical delays against X-rays [1]8]. These values are consistent to the disk size assuming that the light moves from the innermost region to the outer region in the disk.

In addition, we estimated the bolometric luminosity during the terms in which repetitive optical patterns were detected using the Swift/BAT and INTEGRAL IBIS/ISGRI X-ray data. We found 

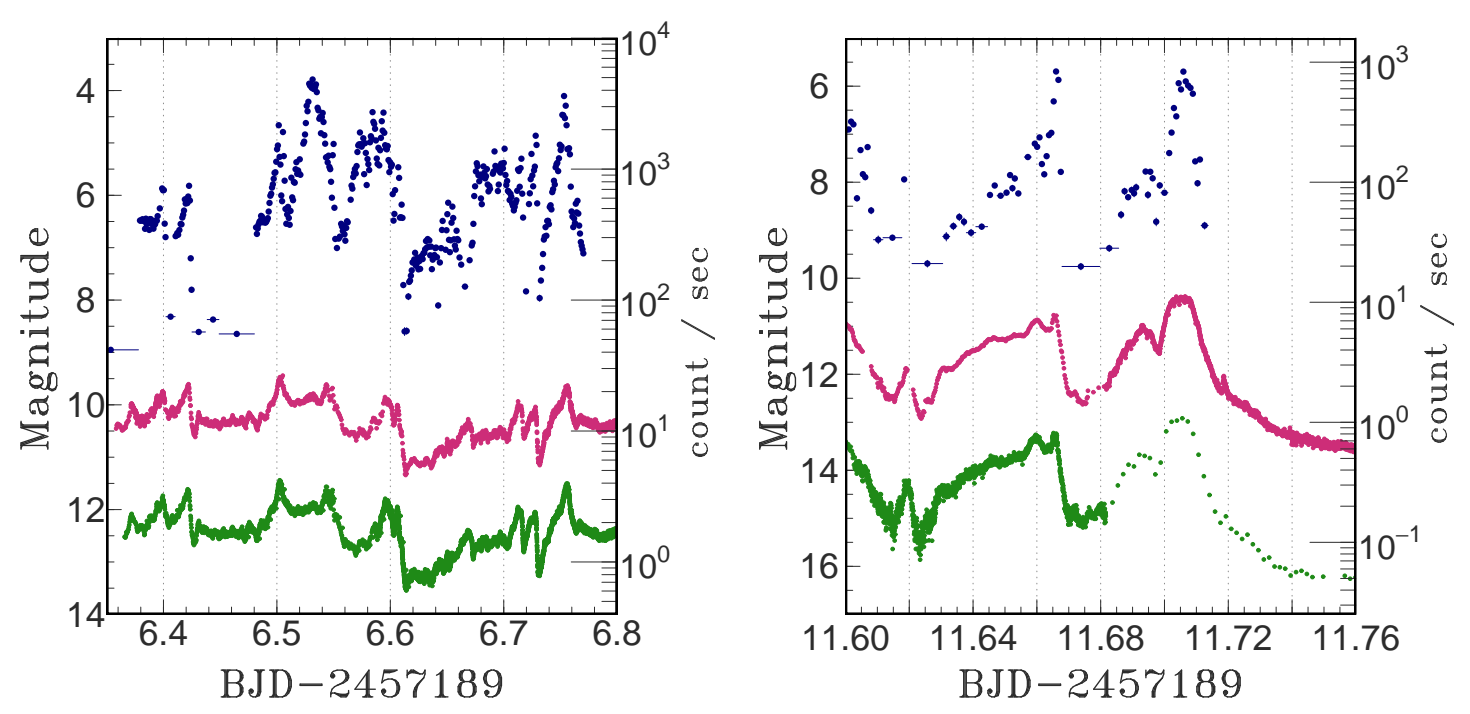

Figure 3: Comparison between optical and X-ray light variations in the June outburst. The navy, pink and green circles represent the INTEGRAL IBIS/ISGRI X-ray data, our $I_{\mathrm{C}}$-band and $V$-band data.

that the dip-type oscillations were observed at low luminosity (less than one tenth of $L_{\mathrm{Edd}}$ ) during the two 2015 outbursts (see Figure 4 in [ㅍ] and Figure 1 in [ㅁ] for the details).

\section{Discussions}

\subsection{Slow Rise and Rapid Decay}

In the two outbursts in 2015, the optical light curves of V404 Cyg showed slow rise and rapid decay, although the rising rate is higher than the fading rate in many outbursts in LMXBs. Slow rise means that the outburst was triggered at the inner region of the accretion disk and it took much time for the heating wave to propagate [BO]].

We estimated the size of the accretion disk lay in hot state in this outburst from the fading rate. The timescale $\tau$ of heating/cooling waves in dwarf novae and X-ray transients is a function of the mass of the central object $\left(M_{1}\right)$ and radius $(r)$ (see equation (7) in [25]]). Substituting the fading rate of the outbursts and the black-hole mass in V404 Cyg into the equation, the estimated value of the outermost disk radius in hot state of V404 Cyg is $\sim 10^{11}$ [cm]. This means that only inner disk lay in hot state in the two outbursts. Actually, our estimates of the disk mass $\left(5 \times 10^{25}[\mathrm{~g}]\right)$ accreted during the June outburst is much smaller than the mass $\left(2 \times 10^{28}\right.$ [g] $)$ of a fully built-up disk in quiescence (This is described in details in Sec. 5 of Methods of [ए7]). The small accreted mass in the June outburst seems to be related to the reason why the December outburst occurred less than 6 months after the June outburst.

\subsection{Origin of Rapid Optical Variations}

Rapid optical variations including dip-type variations which were observed in our photometry would be primarily caused by reprocessing of X-ray irradiation in the disk on the basis of our SED analyses and time lag estimations [ए7, [18]. Prior to the 2015 June outburst in V404 Cyg, the X-ray 
repetitive variations had been observed in other X-ray binaries, GRS 1915+105, IGR J1091-3624 and the Rapid Burster, only with high luminosity, near the Eddington luminosity [ [3], 47], [1]. Hence, existing theories were developing to explain the observations. As for GRS 1915+105, it has been proposed that the variability was caused by bimodal state transitions in the inner accretion disk due to Lightman-Eardley viscous instability which produces slow rises accompanied with sudden decrease in brightness [ $\square]$. However, the dip-type variations in the 2015 June outburst of V404 Cyg were observed even at low luminosity, $\sim 0.01 L_{\text {Edd }}$ [ㄱ] ]. This requires a new explanation about the origin of the repetitive variations observed in X-ray binaries. We proposed that they may be caused by disk properties which are inherent in black-hole binaries with long orbital periods. Actually, the orbital periods of GRS 1915+105, IGR J17091-3624, V4641 Sgr and V404 Cyg, which have showed high-amplitude and short-term variations, are $33.9 \mathrm{~d},>4 \mathrm{~d}, 2.8 \mathrm{~d}$ and 6.5 $\mathrm{d}$, respectively [37, 45, [29, []]. In long-period systems, the surface density seems to be low at the outer disk by viscous diffusion. Actually, the inside-out outbursts characterized by slow rises would indicate the low density at the outer disk in these two outbursts. The continuous mass accretion from the outer part to the inner part of the accretion disk may be difficult due to the low density at the outer disk.

If the violent optical variations with regular patterns were caused by disk properties, an unknown disk instability is likely related to them, which is triggered at low luminosity and cause fluctuations with relatively short timescales ranged from $\sim 10 \mathrm{~min}$ to $\sim 3$ hours. Although its physical origin is still unknown, the suggested new instability triggered at low luminosity could be applicable for other objects containing black holes, such as active galactic nuclei (AGNs). In the case of AGNs, if we assume that the time scales are proportional to the black-hole mass, the time scale of oscillations can be estimated about 10 years. (For example, assuming a massive black hole having $\sim 10^{6} M_{\odot}$, the timescale of the same kind of variations with a timescale of $\sim 100 \mathrm{~min}$ in $\mathrm{X}$-ray binaries having $10 M_{\odot}$ black holes is roughly estimated to be 10 years.) This might provide a new explanation of repetitive variability in AGNs.

\section{Acknowledgements}

We acknowledge the variable star observations from the AAVSO International Database contributed by observers worldwide and used in this research. We also thank the INTEGRAL groups for making the products of the ToO data public online at the INTEGRAL Science Data Centre. This work was supported by a Grant-in-Aid "Initiative for High-Dimensional Data-Driven Science through Deepening of Sparse Modeling" from the Ministry of Education, Culture, Sports, Science and Technology (MEXT) of Japan (25120007 TK). M.K appreciates all observers who belong to VSNET team and collaborators in this work. Many co-authors supported me but I cannot write all members in this paper.

\section{References}

[1] Amnuel, P. R. and Guseinov, O. H. and Rakhamimov, S. J. 1974, Astrophysics \& Space Science, Vol. 29, 331-342

[2] Bagnoli, T., \& in't Zand, J. J. M. 2015, MNRAS, 450, L52 
[3] Belloni, T., Klein-Wolt, M., Méndez, M., van der Klis, M., \& van Paradijs, J. 2000, A\&A, 355, 271

[4] Bernardini, F., Russell, D. M., Shaw, A. W., Lewis, F., Charles, P. A., Koljonen, K. I. I., Lasota, J. P., \& Casares, J. 2016, ApJL, 818, L5

[5] Casares, J., \& Charles, P. A. 1994, MNRAS, 271, L5

[6] Casares, J., Charles, P. A., Naylor, T., \& Pavlenko, E. P. 1993, MNRAS, 265, 834

[7] Chen, W., Shrader, C. R., \& Livio, M. 1997, ApJ, 491, 312

[8] Dubus, G., Hameury, J.-M., \& Lasota, J.-P. 2001, A\&A, 373, 251

[9] Fender, R., \& Belloni, T. 2004, 42, 317

[10] Hynes, R. I., Bradley, C. K., Rupen, M., Gallo, E., Fender, R. P., Casares, J., \& Zurita, C. 2009, MNRAS, 399, 2239

[11] Janiuk, A., \& Czerny, B. 2011, MNRAS, 414, 2186

[12] Jenke, P. A., et al. 2016, ApJ, 826, 37

[13] Jourdain, E., Roques, J.-P., \& Rodi, J. 2016, ArXiv e-prints

[14] Kato, S., Fukue, J., \& Mineshige, S. 2008, )

[15] Kato, T., Uemura, M., Ishioka, R., Nogami, D., Kunjaya, C., Baba, H., \& Yamaoka, H. 2004, PASJ, $56, \mathrm{~S} 1$

[16] Khargharia, J., Froning, C. S., \& Robinson, E. L. 2010, ApJ, 716, 1105

[17] Kimura, M., et al. 2016, Nature, 529, 54

[18] Kimura, M., et al. 2017, MNRAS, 471, 373

[19] Lasota, J.-P. 2001, NewAR, 45, 449

[20] Lehner, M. J., et al. 2009, PASP, 121, 138

[21] Lipunov, V. M., et al. 2016, ApJ, 833, 198

[22] Loh, A., et al. 2016, MNRAS, 462, L111

[23] Makino, F. 1989, IAUcircular, 4782

[24] Martí, J., Luque-Escamilla, P. L., \& García-Hernández, M. T. 2016, A\&A, 586, A58

[25] Meyer, F., 1984, A\&A, 131, 303

[26] Miller-Jones, J. C. A., Jonker, P. G., Dhawan, V., Brisken, W., Rupen, M. P., Nelemans, G., \& Gallo, E. 2009, ApJL, 706, L230

[27] Muñoz-Darias, T., et al. 2016, Nature, 534, 75

[28] Natalucci, L., Fiocchi, M., Bazzano, A., Ubertini, P., Roques, J.-P., \& Jourdain, E. 2015, ApJL, 813, L21

[29] Orosz, J. A., et al. 2001, ApJ, 555, 489

[30] Osaki, Y. 1996, PASP, 108, 39

[31] Rahoui, F., et al. 2016, ArXiv e-prints

[32] Rodriguez, J., et al. 2015, A\&A, 581, L9 
[33] Roques, J. P., \& Jourdain, E. 2016, ArXiv e-prints

[34] Roques, J.-P., Jourdain, E., Bazzano, A., Fiocchi, M., Natalucci, L., \& Ubertini, P. 2015, ApJL, 813, L22

[35] Sanchez-Fernandez, C., Kajava, J. J. E., Motta, S. E., \& Kuulkers, E. 2016, ArXiv e-prints

[36] Shahbaz, T., Ringwald, F. A., Bunn, J. C., Naylor, T., Charles, P. A., \& Casares, J. 1994, MNRAS, 271, L10

[37] Steeghs, D., McClintock, J. E., Parsons, S. G., Reid, M. J., Littlefair, S., \& Dhillon, V. S. 2013, ApJ, 768,185

[38] Tanaka, Y., \& Shibazaki, N. 1996, Annu. Rev. Astron. Astr., 34, 607

[39] Tanaka, Y. T., et al. 2016, ApJ, 823, 35

[40] Tetarenko, A., Sivakoff, G. R., Young, K., Wouterloot, J. G. A., \& Miller-Jones, J. C. 2015, The Astronomer's Telegram, 7708

[41] Tetarenko, B. E., Sivakoff, G. R., Heinke, C. O., \& Gladstone, J. C. 2016, ApJs, 222, 15

[42] Uemura, M., et al. 2002, PASJ, 54, L79

[43] Wagner, R. M., Kreidl, T. J., Howell, S. B., \& Starrfield, S. G. 1992, ApJL, 401, L97

[44] Walton, D. J., et al. 2016, ArXiv e-prints

[45] Wijnands, R., Yang, Y. J., \& Altamirano, D. 2012, MNRAS, 422, L91

[46] Yan, Z., \& Yu, W. 2015, ApJ, 805, 87

[47] Zhang, Z., Qu, J. L., Gao, H. Q., Zhang, S., Bu, Q. C., Ge, M. Y., Chen, L., \& Li, Z. B. 2014, A\&A, 569, A33

[48] Życki, P. T., Done, C., \& Smith, D. A. 1999, MNRAS, 309, 561 